\title{
Lateral lesen. Für eine dezentrierte Hermeneutik der Gegenwart
}

\author{
Klaus Birnstiel
}

Eingegangen: 15. Juli 2021 / Angenommen: 20. Juli 2021 / Online publiziert: 2. November 2021

(C) Der/die Autor(en) 2021

Zusammenfassung Die Probleme hermeneutischer Arbeit in und an der Gegenwart liegen nicht begründet in der Idee des Verstehens selbst, sondern in der Unzulänglichkeit der kommunikationstheoretischen und literatursoziologischen Konzepte hermeneutischer Lektüre. Eine gegenwartsfähige Hermeneutik benötigt ein Update ihrer Kategorien. Hierarchisch gedachte Verhältnisse von Autor, Werk und Lesen sind zugunsten eines breiten, seitwärts verfahrenden Lektürebegriffs zu verabschieden.

Schlüsselwörter Hermeneutik · Gegenwart · Digitalisierung · Lektüre · Autorschaft · Werkbegriff

\section{Reading Laterally. For a Decentered Hermeneutics of the Present}

\begin{abstract}
The problems of hermeneutic work in and on the present are not rooted in the idea of understanding itself, but in the inadequacy of the communication-theoretical and literary-sociological concepts of hermeneutic reading. A hermeneutics capable of dealing with the present needs an update of its categories. Hierarchically conceived relations of author, work, and reading must be abandoned in favor of a broad, laterally proceeding concept of reading.
\end{abstract}

Keywords Hermeneutics · Present · Digitalization · Reading · Authorship · Concept of work 
Ist die Hermeneutik ein Verfahren, oder beschreibt sie ein Verfahren? Am Scheitelpunkt eines hundertfünfzig Jahre währenden Systematisierungs- und Explikationsbemühens, in Hans-Georg Gadamers neo-klassischem Entwurf Wahrheit und Methode von 1960, erscheint sie weder auf die eine noch auf die andere Weise. ${ }^{1}$ Ihre Universalität und Ubiquität lassen sie hier schlicht zum anthropologischen Existential werden: Verstehen ist immer, und so ist auch der Versuch, Verstehen zu verstehen, einer von universalem theoretischen Anspruch. Lesen aber ist eine Praxis, und literaturwissenschaftliches Lesen eine geregelte Praxis, eine Vorgehensweise also, die beschreibbar ist und darüber Nachvollziehbarkeit erreicht. Wer liest, und wer seine Lektüre expliziert, kommt daher nicht umhin, den universalen Anspruch der Hermeneutik in ein partikulares Verfahren zu überführen. Als Verfahren aber hat die Hermeneutik, so scheint es, einen zunehmend schweren Stand.

\section{2}

Betrachtet man die partikulare, literaturwissenschaftliche Geschichte der Hermeneutik, so lässt sich der Eindruck einer Plausibilitätserosion im Methodenstreit des letzten Jahrhunderts kaum abweisen. Überall, wo sich Hermeneutik als Verfahren kritischer Nachfrage ausgesetzt sieht, gerät sie in begriffliche und axiomatische Schwierigkeiten. Andere, im Vokabular wesentlich differenziertere Verfahrensangebote stehen ihr gegenüber. Zusätzlich zum Nachteil gereicht ihr ein doppelter historischer Index. Nicht nur ist die Hermeneutik als Verfahren an heute überwunden geglaubte Epochen der Literaturwissenschaft gebunden - Stichwort >Geistesgeschichte $<-$, sie wird auch, und das ist beiden gegenüber ungerecht, in einen Topf geworfen mit der Praxis der immanenten Interpretation, deren Schwundstufen den immer wieder perhorreszierten Abgrund der Literaturwissenschaft bilden. Darüber hinaus ist die Praxis der hermeneutischen Lektüre besonders gewöhnt an eine klassische, längst historisch gewordene Vorstellung von literarischer Kommunikation. Diese begreift sie als Bewegung im Dreieck von Autor, Werk und Leser, die in einem hierarchischen Verhältnis gedacht werden. Hermeneutisches Lesen bedeutet hier, einem abgeschlossenen Werk den Sinn abzuringen, den ein divinatorisches $\mathrm{Au}-$ torsubjekt auf magische Weise in ihm verborgen hat. Die berühmt läppische Frage, was der Autor uns denn eigentlich habe sagen wollen, ist die treffende Karikatur dieser schlechten Hermeneutik.

\section{3}

Der fachgeschichtliche Index lässt sich nicht tilgen, nur diskutieren. Tatsächlich revidieren aber lässt sich das historisch gewordene literarische Kommunikationsmodell,

\footnotetext{
${ }^{1}$ Gadamer, Hans-Georg: Wahrheit und Methode. Grundzüge einer philosophischen Hermeneutik. Tübingen: Mohr, 1960.
} 
an das sich die literaturwissenschaftliche Hermeneutik so schmiegsam gebunden hat. Dass dazu Anlass und Grund bestehen, zeigt unter anderem der Blick auf die allerneueste Gegenwartsliteratur. Schreibweisen der Gegenwart nach der Digitalisierung haben statt in Zwischenräumen von Buchkultur und sozialen Medien. ${ }^{2}$ Ihre Produktion wie Rezeption vollzieht sich im Gebrauch digitaler Applikationen. Dazu gehört auch die Literaturkritik, die sich ebenfalls zunehmend im digitalen Raum abspielt, beziehungsweise dort selbst einen kritischen Echoraum findet. Wer den komplexen Linien dieser Literaturen folgen und sie verstehen will, ist auf eine Lektürepraxis angewiesen, die den konzentrierten Leseblick zumindest zeitweise von der Buchseite löst und in die Welt um das Buch herum eintaucht. Ein solches Lesen nimmt die Feuilletons und sonstigen Medienbeiträge zur Kenntnis, es studiert Interviews und andere Aktivitäten von Akteurinnen und Akteuren des Literaturbetriebs, und lässt sich auf die Spiele mit Produktions-, Rezeptions- und Sinnebenen ein, die sich in den sozialen Medien ergeben. Was einst Paratext genannt wurde, was Epitext, was Kritik, ist nun Teil des Textes der Literatur selbst, und will als solcher und solche gelesen werden.

\section{4}

>Literatur ist hier nicht mehr zu verstehen als gebunden an drucktechnische und andere Formatierungen, sondern als ästhetisches Ereignis in je spezifischer Gestalt. Medienmischung ist ein konstitutives Element. Virtuos überschreitet die »ubiquitäre Literatur « (Holger Schulze) die Grenzen von Realwelt und Diegese, Figur und Autor, Text und Paratext, Werk und Kommentar. ${ }^{3}$ Wer sich durch diesen Dschungel bewegt, verlässt den Bannkreis des gedruckten Buches - er liest, und er liest seitwärts: neben das Buch treten die veröffentlichten Kritiken, das Rauschen im Blätterwald, die Kommentare in Funk und Fernsehen, die Verlautbarungen der Verlage und der Literaturbetrieb-Insider, und nicht zuletzt die Performances der Autorinnen und Autoren. Der ästhetische Genuss und intellektuelle Gewinn ergibt sich aus eben dieser seitwärtigen, lateralen Lesebewegung weg vom Buch, hinein in die Literatur. Seitwärts, lateral lesen heißt demnach, sich auf die intermedialen Effekte und Aspekte von Gegenwartsliteratur einzulassen. Literaturwissenschaftlich lateral zu lesen heißt, die Bedingungen und Effekte des zugrundeliegenden Wandels zu studieren und für die literaturwissenschaftliche Begriffsarbeit entsprechende Konsequenzen zu ziehen.

\footnotetext{
2 Die Formulierung von den »Schreibweisen der Gegenwart nach der Digitalisierung « entspricht dem Titel eines DFG-geförderten Greifswalder Forschungsprojekts unter der Leitung von Eckhard Schumacher. Ihm, Elias Kreuzmair und Magdalena Pflock sei für die Etablierung einer steten Anregung im Nahbereich gedankt. Ebenso zu danken ist Johannes Franzen und Eva Stubenrauch in Bonn, wo eine erste Fassung dieser Überlegungen im Herbst 2020 präsentiert und diskutiert werden konnte.
}

3 Schulze, Holger: Ubiquitäre Literatur. Eine Partikelpoetik. Berlin: Matthes \& Seitz, 2020. 
Es ist selbstredend alles andere als überraschend, dass Autorinnen und Autoren sich in zunehmendem Maße sogenannter neuer Medien bedienen, und wer mag, kann diese Einsätze einfach als zeitgemäße flankierende Buchmarkt-Maßnahmen lesen. Die Empfehlung, lateral, seitwärts zu lesen, kann aber abgestützt werden auf so etwas wie eine >Theorie< der Gegenwart, die, wie es scheint, selbst zunehmend lateral organisiert ist. Von dort aus gesehen, wird der Aufruf zum lateralen Lesen womöglich eine etwas größere Plausibilität erlangen. Wer über Gegenwart spricht und schreibt, kommt um bestimmte Schlagwörter nicht herum, namentlich nicht um das von der Digitalisierung. Zeitdiagnostische Schriften zu den Effekten der sogenannten >Digitalisierung sind allerdings selten haltbarer als eine Buchmarktsaison; schon nach wenigen Jahren erscheinen sie als in geradezu rührender Weise veraltet. $\mathrm{Zu}$ groß, zu klein: die kulturkritische Gegenwartsdiagnostik leidet an einem Form-Problem, das zugleich ein Strukturproblem ist. Großtheorien können hier nur dann Plausibilität erlangen, wenn sie in Diagnose-Optiken mit mittlerer Reichweite umgebaut werden können. Möglich ist dies mit einem Denkangebot, das Hans Ulrich Gumbrechts 2010 erschienene kleine Schrift Unsere breite Gegenwart unterbreitet. ${ }^{4}$ Hierin und in einer Reihe zugehöriger verstreuter Publikationen verknüpft Gumbrecht, der sich bekanntermaßen seit den 1980er Jahren an Begriffen wie Postmoderne, Posthistoire und Ähnlichen abgearbeitet hatte, eine Reihe von eher lose verfugten Gegenwartsbeobachtungen mit einer geschichtsphilosophischen Deutung. Zentral ist die Feststellung, dass die digitale Transformation der Gesellschaft nicht nur räumliche, sondern auch zeitliche Distanzen hat schwinden lassen: nicht nur ist es möglich, mithilfe digitaler Medien, das heißt insbesondere der globalen Integrationsmaschine $>$ Internet $<$, räumlich weit entfernte Äußerungen und Diskurse als benachbart wahrzunehmen und sich an ihnen zu beteiligen. Auch die zeitliche Entfernung relativiert sich. Elektronische Medien und Archive halten Vergangenes ständig in einer Weise präsent und verfügbar, die noch vor wenigen Jahrzehnten undenkbar erschien. Gumbrecht ergänzt diese Beobachtungen der digitalen Gegenwart um eine geschichtsphilosophische Spekulation. Dieser Spekulation zufolge erleben wir seit den siebziger Jahren des 20. Jahrhunderts, getrieben vom digitalen Wandel, eine Veränderung unseres Verhältnisses zur Zeit an und für sich: Gumbrecht spricht hier vom »Wandel der Chronotopen «. ${ }^{5}$ In der historischen Zeit, der Zeit des 19. Jahrhunderts, sind wir es gewohnt, Vergangenheit beständig hinter uns zu lassen und mit Siebenmeilenstiefeln einer offenen Zukunft entgegenzuschreiten. Die Gegenwart selbst erscheint dabei als minimal kurzer Augenblick des Übergangs von Vergangenheit in Zukunft. Versuchen wir die Gegenwart zu fassen, so ist sie bereits wieder vergangen, und eine weitere, weite Zukunft lichtet sich vor uns. >Mitnehmen< können wir allenfalls die transzendenten,

\footnotetext{
${ }^{4}$ Gumbrecht, Hans Ulrich: Unsere breite Gegenwart. Aus dem Englischen von Frank Born. Berlin: Suhrkamp, 2010. Gumbrechts dezidiert anti-hermeneutische Position (Ders.: Diesseits der Hermeneutik. Die Produktion von Präsenz. Übersetzt von Joachim Schulte. Frankfurt a. M.: Suhrkamp, 2004) wäre mit dem hier Vorgebrachten zumindest in Teilen durchaus zusammenzubringen, bleibt aber außer Acht.

5 Gumbrecht, Hans Ulrich: »Vom Wandel der Chronotopen. Ein mögliches Nachwort.« In: Ders./Erik Schilling (Hg.): Literatur und Theorie seit der Postmoderne. Stuttgart: Hirzel, 2012, S. 229-236.
} 
hermeneutisch herauspräparierten Sinngehalte der Vergangenheit, herausgelöst aus ihren Ursprungskontexten. Irgendwann im 20. Jahrhundert, nach zwei Weltkriegen und anderen planetaren Katastrophen, zerfällt dieses geschichtsteleologische Modell, und eine neue Konfiguration der drei Zeiten Vergangenheit, Gegenwart und Zukunft tritt hervor. Während Vergangenheiten nicht mehr einfach vergehen, sondern von einem öffentlichen Erinnerungsdiskurs beständig präsent gehalten werden, erscheint uns die Zukunft nicht mehr als offener Horizont von Möglichkeiten, aus denen wir auswählen können, sondern als verschlossen - verschlossen dank planetarer Katastrophenszenarien wie Weltwirtschaftskrise oder Erderwärmung, die wir kommen sehen, aber nicht abwenden können. Resultat dieser Verschiebungen ist, dass sich die Gegenwart verändert: von dem unmittelbar kurzen Moment des Übergangs, dieser sogleich vergehenden Sekunde, wird sie zu einem Raum der Simultaneitäten, in welchem verschiedenste Phänomene, vergangene, zukünftige und gegenwärtige, ko-präsent sind. Die Gegenwart dehnt sich also in beide Richtungen des Zeitstrahls aus, sie wird $>$ breiter $<$.

\section{6}

Die durch digitale Techniken Medien induzierte gesellschaftliche Transformation hin zu einer breiten Gegenwart lässt sich auch als Prozess der Lateralisierung begreifen: entfernte Gegenstände sind nicht mehr entfernt, Ereignisse, Diskurse und Positionen finden sich nicht vor oder hinter uns, sondern stets neben uns, seitlich von uns. Die Verweisarchitektur von Online-Medien ist dafür ein fast zu gutes Beispiel. Mit wenigen Klicks ist es möglich, sich seitwärts durch eine ganze Reihe von Diskursen zu bewegen. Zeitliche und räumliche Distanz verschwinden zwar nicht zur Gänze, werden in ihrer Bedeutung aber reduziert. Die breite Gegenwart erscheint so als ein Raum lateraler Verknüpfungen, durch den man sich seitwärts, nicht vorwärts oder rückwärts bewegt. Dahingestellt, ob Lateralisierung nun Vorschein einer postkapitalistischen Gleichheitsutopie oder aber nur der jüngste Trick des Spätkapitalismus ist, so lässt sich mit einiger Vorsicht zumindest behaupten, dass die Lateralisierung der gesellschaftlichen Verhältnisse insgesamt zu einer gewissen Enthierarchisierung führt.

\section{7}

Wenn der Haupteffekt der Lateralisierung gesellschaftlicher Verhältnisse in ihrer Enthierarchisierung besteht, so lässt sich solches auch mit Blick auf den Werkcharakter der Kunst sowie ihre Situierungs- und Rezeptionsverhältnisse vermuten. Die soziale Distanz zum Kunstwerk schwindet ebenso wie die Distanz vom Kunstwerk zum Nicht-Kunstwerk. Die Entteleologisierung der Geschichte der Gegenwart eröffnet einen breiten Raum kurzlebigerer Sinnstiftungsvereinbarungen, welche oftmals nicht ideenförmiger, sondern ästhetischer Natur sind. Ästhetische Praktiken werden Teil der Lebenswelt, und die Sonderzuständigkeit der artefaktorientierten Kultur- und Kunstwissenschaften verringert sich entweder auf Spezialistendiskurse 
zu ästhetischen Partikularphänomenen oder aber weitet sich auf das große gesellschaftliche Ganze. Eine griffige Formulierung Peter Osbornes bringt den Wandel auf den Punkt. Aus seinen Studien zur Kunst nach dem Ende der Konzeptkunst der sechziger Jahre und danach ergibt sich für Osborne die plakative Feststellung, dass die Kunst der Gegenwart »anywhere or not at all « ist (oder zu sein hat). ${ }^{6}$ Kunst, die sich selbst auf ihre Existenz im white cube beschränkt, ist ästhetisch und gesellschaftlich obsolet, und ein Kunstbegriff, der Kunst in einem absoluten Außerhalb ästhetischer Selbstschöpfung beheimatet sieht, hat zu den experimentellen, inter- und transmedialen Praktiken von Gegenwartskunst nichts zu sagen. Was für die Kunst gilt, kann aber auch für die Literatur und schließlich für ihre wissenschaftliche Erforschung in Anschlag gebracht werden. Gerade im Blick auf die Literatur der Gegenwart kann, ja muss der goethezeitliche Plunder von der Autonomie des Kunstwerks, seiner ingeniösen Emanation aus der Autorpersönlichkeit und seiner tiefenhermeneutischen Rezeption durch versenkende Lektüre beiseite geräumt werden. Gegenwartsliteratur entsteht und lebt in vielfältigen transmedialen, institutionellen und individuellen Zusammenhängen; wer sich mit ihr angemessen beschäftigen will, muss sich lateral lesend durch sie hindurchbewegen und seine oder ihre alten Zopfkategorien von Autor, Werk und Lesen im Universitätsarchiv abgeben.

\section{8}

Ein solches, gegenwartsfähiges Update der Literaturwissenschaft erfindet die Hermeneutik nicht neu. Es ordnet aber die Analysefelder institutionen- und begriffstheoretisch so um, dass die Möglichkeit zu hermeneutischem Verstehen überhaupt erst wieder aufscheint. Nur die konsequente Historisierung der literaturwissenschaftlichen Basiskategorien macht diese wieder gegenwartsfähig. Ein Begriff von gegenwärtiger Autorschaft etwa, der nicht auf die medialen Bedingungen von Autorschaft im Zeitalter von Twitter, Facebook \& Co. reflektiert, ist ebenso obsolet wie ein Textbegriff, der sich von der impliziten Normativität der Druckseite nicht zu lösen vermag. Sämtliche literaturwissenschaftliche Grundbegriffe sind in medialer Hinsicht zu reflektieren. Erst dann werden sie zu brauchbaren, das hermeneutische Verständnis leitenden Rahmenannahmen. In der breiten Gegenwart mit ihrer Vorherrschaft digital tingierter Lebenswelten, in einem zunehmend enthierarchisierten Literatursystem, in welchem die goethezeitlichen Ordnungen und ihre institutionelle Autorität verblassen, scheint es insgesamt angezeigt, auf laterale Lesestrategien zu setzen - auf Lesestrategien also, die die obskure Divinatorik der älteren Hermeneutik und ihres bösen Zwillings, der Dekonstruktion, ersetzen durch eine agile Lesebewegung der seitwärtigen Orientierung und Anschlussbildung. Zurecht erntet die Rede von der angeblich gebotenen Trennung von Autor und Werk in der kulturkritischen Netzöffentlichkeit nurmehr Hohn und Spott, wenn wieder einmal ein Autor seine schwindende Medienmacht zur Verbreitung rechtspopulistischen Gefasels nutzt. Sie ist ein heuristisches Phantasma, das für alles Mögliche von Nutzen ist, nicht aber für das Reden über Gegenwart und Gegenwartsliteratur. Lateral zu lesen bedeu-

\footnotetext{
${ }^{6}$ Osborne, Peter: Anywhere Or Not At All. Philosophy of Contemporary Art. London: Verso, 2013.
} 
tet, die hergebrachte Ordnung von Autor, Werk und Leser in ihrer hierarchischen Eindeutigkeit nicht mehr zu akzeptieren. »Autorschaft ist Werkherrschaft«, lautete die von Heinrich Bosse geprägte, klassische, und für die Klassik richtige, Formulierung zu künstlerischer Autonomie und ästhetischem Eigentum. ${ }^{7}$ Die Herrschaft über das Werk bestimmt nicht nur dessen konkrete Gehalte, sondern vor allem legt sie eindeutig fest, wo ein Werk beginnt und endet, was zum Werk gehört und was nicht. Literarisches Paradigma war, mediengeschichtlich gewendet, das Buch, mit Einband, Titelei, Anfang und Ende. Im Lichte einer lateral organisierten Gegenwart aber sind solche Autorschafts- und Werkbegriffe erneut und radikal infrage zu stellen. Das Werk wird darüber zum Text, der nach verschiedenen Seiten hin offen ist und geöffnet werden kann.

\section{9}

Sind Autor und Werk in ihr laterales Jetzt aufgelöst, folgt nun noch einmal, als allerletzter Hakenschlag, die Leserin, der Leser, das Lesen. Es wurde schon angedeutet, dass jede Literaturwissenschaft eine Kontextwissenschaft im breitesten Verständnis sein muss. Wenn Kunst, wenn Literatur »anywhere or not at all« sind, dann muss auch das Lesen »anywhere or not at all« sein, losgelöst von der Fixierung auf die Buchseite, bereit zu lateraler Bewegung durch den Text, die Texte, und weit über den Text hinaus. An die Seite der ubiquitären Literatur der Gegenwart stellt sich so ein laterales Lesen, und damit eine ubiquitäre Hermeneutik.

Funding Open Access funding enabled and organized by Projekt DEAL.

Open Access Dieser Artikel wird unter der Creative Commons Namensnennung 4.0 International Lizenz veröffentlicht, welche die Nutzung, Vervielfältigung, Bearbeitung, Verbreitung und Wiedergabe in jeglichem Medium und Format erlaubt, sofern Sie den/die ursprünglichen Autor(en) und die Quelle ordnungsgemäß nennen, einen Link zur Creative Commons Lizenz beifügen und angeben, ob Änderungen vorgenommen wurden.

Die in diesem Artikel enthaltenen Bilder und sonstiges Drittmaterial unterliegen ebenfalls der genannten Creative Commons Lizenz, sofern sich aus der Abbildungslegende nichts anderes ergibt. Sofern das betreffende Material nicht unter der genannten Creative Commons Lizenz steht und die betreffende Handlung nicht nach gesetzlichen Vorschriften erlaubt ist, ist für die oben aufgeführten Weiterverwendungen des Materials die Einwilligung des jeweiligen Rechteinhabers einzuholen.

Weitere Details zur Lizenz entnehmen Sie bitte der Lizenzinformation auf http://creativecommons.org/ licenses/by/4.0/deed.de.

\footnotetext{
7 Bosse, Heinrich: Autorschaft ist Werkherrschaft. Über die Entstehung des Urheberrechts aus dem Geist der Goethezeit. Paderborn/München u. a.: Schöningh 1981.
} 